\title{
Medicinal plants used in the Amazon region: a systematic review
}

\author{
Plantas medicinais utilizadlas na região Amazônica: uma revisão sistemática \\ Plantas medicinales utilizadas en la región amazónica: una revisión sistemática
}

Received: 28/28/2021 | Reviewed: 09/02/2021 | Accept: 10/27/2021| Published: 10/29/2021

\author{
João Galdino de Páscoa Júnior \\ ORCID: https://orcid.org/0000-0001-6516-3626 \\ Universidade Estadual de Roraima, Brazil \\ E-mail: joao.pascoa@hotmail.com \\ Iara Leão Luna de Souza \\ ORCID: https://orcid.org/0000-0002-5309-2979 \\ Universidade Estadual de Roraima, Brazil \\ Centro Universitário Estácio da Amazônia, Brazil \\ E-mail: iaraluna@uerr.edu.br
}

\begin{abstract}
The Amazon region's biodiversity is exploited by the local population, commonly for therapeutic purposes. Given this information, the goal of this study was to perform a systematic review on medicinal plants used in this region, listing the name of the species, location found, and its importance for traditional medicine and for local people. For this, a search was performed in the PubMed/Medline and Lilacs databases, using the descriptors medicinal plants, Amazonia or Amazon region, in Portuguese, English, and Spanish. The main findings demonstrate that different species from different botanical families are used by the local population. The main therapeutic outcomes sought out refer to the search for relief or treatment of gastrointestinal, hepatic alterations, fever, kidney stones, pain, infections, and parasites. Thus, it is concluded that the practice of traditional medicine is very common in the Amazon region and is transmitted orally from generation to generation. In this practice, the use of medicinal plants in the form of tea is highlighted, with the species in the form of powder, dried or natural plants. Therefore, it is recommended that the strengthening of pharmacological studies involving local medicinal plants can provide scientific support for popular knowledge.
\end{abstract}

Keywords: Amazonia; Traditional medicine; Folk knowledge; Ethnopharmacology.

\section{Resumo}

A região amazônica possui uma grande biodiversidade, esta que é explorada pela população local, comumente, com a finalidade terapêutica. Diante dessas informações, o objetivo deste estudo foi realizar uma revisão sistemática sobre as plantas medicinais utilizadas nessa região, listando os nomes das espécies, o local encontrado e a sua importância para a medicina tradicional e para a população local. Para isso, realizou-se uma busca nos bancos de dados PubMed/Medline e Lilacs, utilizando os descritores plantas medicinais, Amazônia ou região amazônica, nos idiomas português, inglês e espanhol. Os principais achados demonstram que diferentes espécies de variadas famílias botânicas são utilizadas pela população local. Os principais desfechos terapêuticos buscados se referem à busca do alívio ou tratamento de alterações gastrointestinais, hepáticas, febre, cálculos renais, dor, infecções e parasitoses. Diante disso, conclui-se que a prática da medicina tradicional é muito usual na região amazônica e transmitida de forma oral de geração para geração. Nessa prática, destaca-se o uso de plantas medicinais na forma de chá, estando as espécies na forma de pó, plantas secas ou naturais. Logo, recomenda-se o fortalecimento dos estudos farmacológicos envolvendo as plantas medicinais locais pode fornecer respaldo científico para o conhecimento popular.

Palavras-chave: Amazônia; Medicina tradicional; Conhecimento popular; Etnofarmacologia.

\section{Resumen}

La región amazónica tiene una gran biodiversidad, que es comúnmente explorada por la población local con fines terapéuticos. Ante esta información, el objetivo de este estudio fue realizar una revisión sistemática de las plantas medicinales utilizadas en esta región, enumerando el nombre de la especie, la ubicación encontrada y su importancia para la medicina tradicional y para la población local. Para ello, se realizó una búsqueda en las bases de datos PubMed/Medline y Lilacs, utilizando los descriptores plantas medicinales, Amazonia o Región Amazónica, en portugués, inglés y español. Los principales hallazgos demuestran que la población local utiliza diferentes especies de diversas familias botánicas. Los principales resultados terapéuticos buscados se refieren a la búsqueda de alivio o tratamiento de trastornos gastrointestinales y hepáticos, fiebre, cálculos renales, dolores, infecciones y parásitos. Por tanto, se concluye que la práctica de la medicina tradicional es muy común en la región amazónica y se transmite por vía oral de generación en generación. En esta práctica se destaca el uso de plantas medicinales en forma de té, con las 
especies en forma de polvo, plantas secas o naturales. Por lo tanto, se recomienda que el fortalecimiento de los estudios farmacológicos que involucren plantas medicinales locales pueda brindar soporte científico al conocimiento popular.

Palabras clave: Amazonia; Medicina tradicional; Saberes populares; Etnofarmacología.

\section{Introduction}

The Amazon Rainforest is known as the largest forest on Earth and for sheltering the largest river basin in the world. It is distributed by nine countries in South America, with the largest portion in Brazilian lands (67.8\%), where it is called Legal Amazon and it comprises the states of Acre, Amapá, Amazonas, Maranhão, Mato Grosso, Pará, Rondônia, Roraima and Tocantins (Penna-Filho, 2013).

In addition to all its territory greatness, the Amazon has nine kinds of vegetation, of which Terra Firme Forest is the most important, since it covers $90 \%$ of the Legal Amazon and presents great variation of plant species that were not or are little explored (Braga, 1979; Penna-Filho, 2013), having them a great potential for generating resources, such as those related to the pharmaceutical and medical areas.

The potential of using medicinal plants as a treatment for diseases, called phytotherapy, is a practice of traditional medicine that has been used since the appearance of the human species (Badke et al., 2011), however, over the years and with the advance of technology, new ways to treat diseases were being developed and the use of medicinal plants became less common. Nevertheless, phytotherapy practice in Brazil has not lost its relevance despite the great incentives of the pharmaceutical industry in the country, due to folk knowledge about medicinal plants (Badke et al., 2011).

Additionally, with the advancement of Brazilian health models until the arrival of the Unified Health System, it can be understood that medicinal plants and herbal medicines have a lot to add in developing countries, as they are important instruments of pharmaceutical care, since $67 \%$ of medicinal plant species in the world originate from developing countries. Since the past, several notices and resolutions of the World Health Organization (WHO) express the organization's position regarding the need to value the use of these drugs, in the health field, being observed as a normative basis for the maintenance of health (Unesco, 2020).

In 2006, the National Policy on Integrative and Complementary Practices was granted in order to establish guidelines and institutional responsibilities for the implementation/adaptation of actions and services in traditional Chinese medicine/acupuncture, homeopathy, medicinal plants and phytotherapy, in addition to establishing health observatories for social thermalism/crenotherapy and for anthroposophical medicine in the Unified Health System (Brasil, 2012).

In line with the above, in 2012, the Brazilian Ministry of Health launched the Primary Care Booklet 31, which guides the actions to be performed by the Basic Health Units and their professionals, in addition to making a historical overview of national policies and informing on the norms, guidelines, services and products related to phytotherapy in the Family Health Strategy/Primary Care. The objective of this is to implement new programs, improving the population's access to safe and quality products and services; sensitize and guide managers and health professionals in the formation and implementation of policies, programs, projects and structures that aim to strengthen phytotherapy, with an emphasis on primary care (Brasil, 2012).

In this context, since the Amazon Rainforest is a rich region with a large number of plants and phytotherapy practice is relevant in Brazil, the goal of this study was to perform a systematic review on medicinal plants used in this region, listing the name of the species, location found, and its importance for traditional medicine and for local people.

\section{Methodology}

The study is a systematic review of scientific articles on medicinal plants found in the Amazon region, which are used 
in folk medicine; and it was made following the recommendation for PRISM reports of systematic reviews and meta-analyses (Moher et al., 2009).

Eligible studies were identified in searches carried out on July 4, 2019, in the PubMed/Medline (National Library of Medicine, Bethesda, MD) and Lilacs (Latin American and Caribbean Literature on health sciences) databases. The keyword combinations used in database searches, covering the Portuguese, Spanish and English languages, used in accomplishment researches were ("Plantas medicinais") AND (“Amazonia OR região amazonica"), (“Plants medicinal”) AND (“Amazon OR amazon region”) and (“Plantas medicinales") AND (“Amazon OR región amazónica”). In this research, all items were identified, regardless of study design.

The criteria for inclusion were data about the medicinal plants of the Amazon region, which are used by folk medicine for the treatment of diseases. Additionally, the exclusion criteria used were divided into three categories. First, data with a publication period before 2009. Second, review articles, historical documents, theses, and dissertations, considering only scientific articles. Third, escape from the theme of the present study, as well as data published without location information, locations outside of the Amazon region, lack of scientific names of medicinal plants and data that does not demonstrate the use of medicinal plants by population, with based on reading the title and abstract of the article.

The selected articles were evaluated by the reviewers considering the reading and careful analysis of the full text, by removing those who deliberate the exclusion criteria adopted. Still, the existence of data duplication in the same database and between databases was checked, and each item was counted only once. The information to be removed from articles included in the study were the names of plant species, the reason for its use, and how it was used, according to the Amazon region.

The results were described in a table, listing the characteristics of the botanical family, the plant species, the purpose of the study, the medicinal use, the form of preparation of the medicinal plant, the route of administration, the location found and the authors and year of the articles (Table 1).

\section{Results}

After the use of the above descriptors in the databases, 311 published articles were identified between January 1, 2009, and the date of search (July 4, 2019), of which 39 were repeated and were counted only once. After deleting them, there were 272 remaining items, of which 36 were not scientific articles. Then, reading the titles and summaries of other 236 articles, to identify which did not fit the criteria for inclusion, data about the medicinal plants of the Amazon region, which are used by folk medicine for the treatment of diseases. After this analysis, 51 items remained. With the reading of the articles in their entirety, there were 13 items left which were included in this review, because the others didn't bring the information about the medicinal plants of the Amazon region, which are used by folk medicine for the treatment of diseases (Figure 1).

The results obtained regarding the botanical family, the main species, the purposes of the studies, the medicinal use, the form of use and the local application of medicinal plants in the Amazon region are listed in Table 1. 
Figure 1 - Flowchart for the systematic process of article selection according to the PRISMA model.

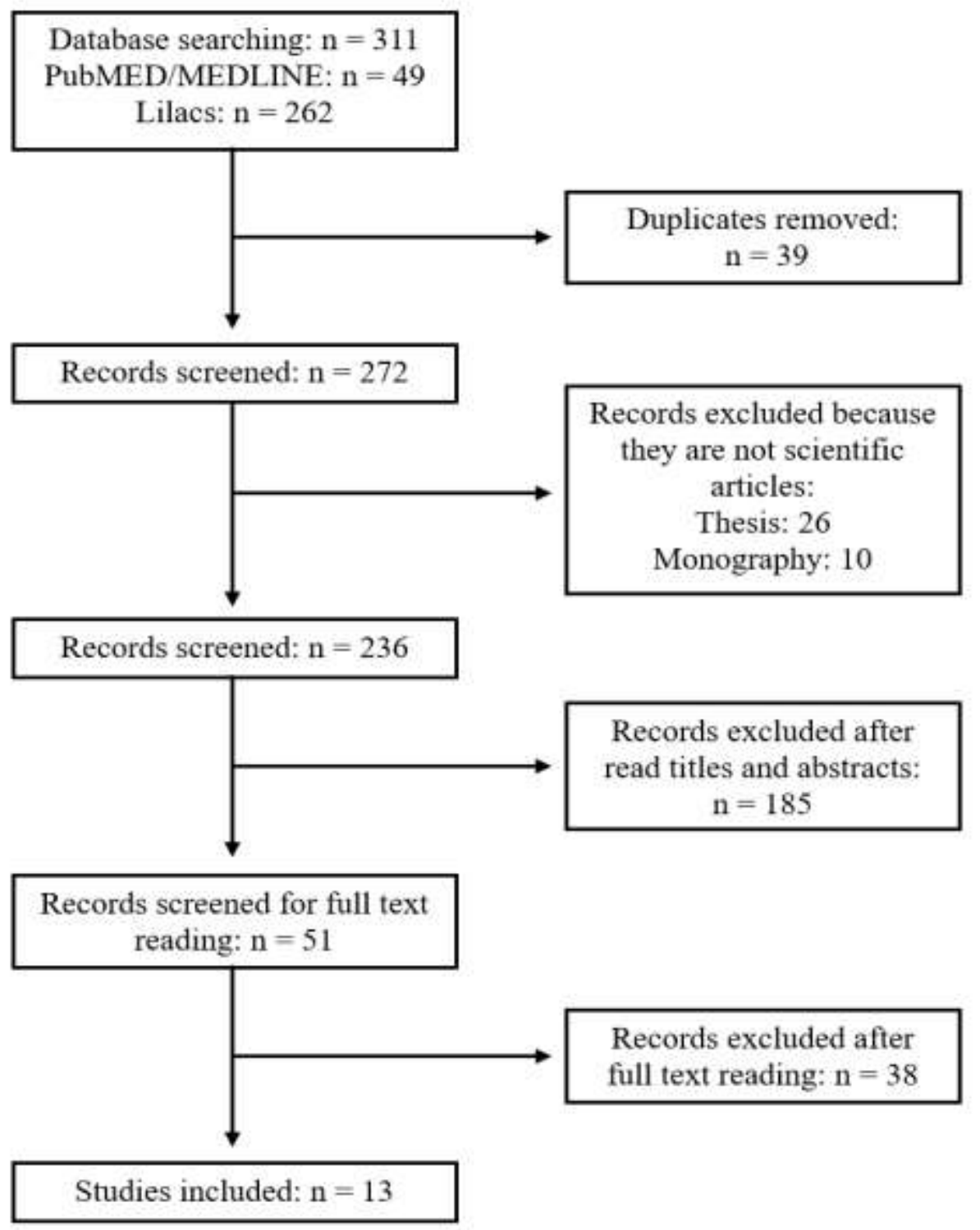

Source: Authors. 
Table 1 - Characteristics of studies on the use of medicinal plants by the Amazon region population published between the years of 2009 and 2019.

\begin{tabular}{|c|c|c|c|c|c|c|c|}
\hline $\begin{array}{c}\text { Botanical } \\
\text { family }\end{array}$ & Main species & Purpose of the study & Medicinal use & Preparation & $\begin{array}{c}\text { Route of } \\
\text { administration }\end{array}$ & Place & $\begin{array}{c}\text { Author, } \\
\text { year }\end{array}$ \\
\hline \multirow{8}{*}{ Fabaceae } & Erythrina fusca Loureiro & $\begin{array}{c}\text { Studying, recording and } \\
\text { disseminating ethno-knowledge }\end{array}$ & $\begin{array}{l}\text { Tumors, } \\
\text { inflammation and } \\
\text { cancer }\end{array}$ & Cooked & Oral & $\begin{array}{l}\text { Brazil- } \\
\text { Colombia- } \\
\text { Peru } \\
\text { Border }\end{array}$ & $\begin{array}{l}\text { Rengifo- } \\
\text { Salgado et } \\
\text { al., } 2017\end{array}$ \\
\hline & $\begin{array}{l}\text { Diplotropis purpurea var. } \\
\text { leptophylla (Kleinhoonte) } \\
\text { Amshoff }\end{array}$ & $\begin{array}{c}\text { Studying, recording and } \\
\text { disseminating ethno-knowledge }\end{array}$ & Tonsillitis & Infusion & Oral & $\begin{array}{l}\text { Brazil- } \\
\text { Colombia- } \\
\text { Peru } \\
\text { Border }\end{array}$ & $\begin{array}{l}\text { Rengifo- } \\
\text { Salgado et } \\
\text { al., } 2017\end{array}$ \\
\hline & $\begin{array}{c}\text { Capaifera paupera }(\text { Herzog }) \\
\text { Dwyer }\end{array}$ & $\begin{array}{c}\text { Studying, recording and } \\
\text { disseminating ethno-knowledge }\end{array}$ & $\begin{array}{l}\text { Gastritis and } \\
\text { inflammation }\end{array}$ & Direct use & Oral and topic & $\begin{array}{l}\text { Brazil- } \\
\text { Colombia- } \\
\text { Peru } \\
\text { Border }\end{array}$ & $\begin{array}{l}\text { Rengifo- } \\
\text { Salgado et } \\
\text { al., } 2017\end{array}$ \\
\hline & $\begin{array}{l}\text { Campsiandra angustifolia } \\
\text { Spruce ex Bentham }\end{array}$ & $\begin{array}{c}\text { Studying, recording and } \\
\text { disseminating ethno-knowledge }\end{array}$ & $\begin{array}{l}\text { Diarrhea, "belly" } \\
\text { pain, liver and } \\
\text { rheumatism }\end{array}$ & Cooked & Oral & $\begin{array}{l}\text { Fronteira } \\
\text { Brasil- } \\
\text { Colombia- } \\
\text { Perú }\end{array}$ & $\begin{array}{l}\text { Rengifo- } \\
\text { Salgado et } \\
\text { al., } 2017\end{array}$ \\
\hline & $\begin{array}{l}\text { Vigna unguiculata }(\mathrm{L} .) \\
\text { Walpers }\end{array}$ & $\begin{array}{c}\text { Studying, recording and } \\
\text { disseminating ethno-knowledge }\end{array}$ & Diuretic and laxative & Cooked & Oral & $\begin{array}{l}\text { Fronteira } \\
\text { Brasil- } \\
\text { Colombia- } \\
\text { Perú }\end{array}$ & $\begin{array}{l}\text { Rengifo- } \\
\text { Salgado et } \\
\text { al., } 2017\end{array}$ \\
\hline & Senna alata (L.) Roxburgh & $\begin{array}{c}\text { Studying, recording and } \\
\text { disseminating ethno-knowledge }\end{array}$ & $\begin{array}{l}\text { Antibacterial, } \\
\text { diarrhea and } \\
\text { infections }\end{array}$ & Infusion & Oral & $\begin{array}{c}\text { Fronteira } \\
\text { Brasil- } \\
\text { Colombia- } \\
\text { Perú } \\
\text { Santa }\end{array}$ & $\begin{array}{l}\text { Rengifo- } \\
\text { Salgado et } \\
\text { al., } 2017\end{array}$ \\
\hline & Anadenanthera peregrina & $\begin{array}{c}\text { Seek information from indigenous } \\
\text { communities about medicinal plants } \\
\text { used as anti-malarials }\end{array}$ & Malaria & Inhalation & ------- & $\begin{array}{l}\text { Isabel do } \\
\text { Rio } \\
\text { Negro, } \\
\text { Brazil }\end{array}$ & $\begin{array}{c}\text { Frausin et } \\
\text { al., } 2015\end{array}$ \\
\hline & $\begin{array}{l}\text { Amburana cearensis } \\
\text { (Fr.Allem.) A.C.Sm. }\end{array}$ & $\begin{array}{l}\text { Describe traditional medicine and } \\
\text { analyze plants that can be } \\
\text { investigated pharmacologically }\end{array}$ & Diabetes and anemia & Decoction & Intern use & $\begin{array}{l}\text { Porvenir, } \\
\text { Bolivia }\end{array}$ & $\begin{array}{c}\text { Hajdu and } \\
\text { Hohmann } \\
2012\end{array}$ \\
\hline \multirow[t]{2}{*}{ Arecaceae } & Dalbergia monetária L.f & $\begin{array}{l}\text { Select species for the treatment of } \\
\text { gastrointestinal disorders }\end{array}$ & Diarrhea & ------- & ------- & $\begin{array}{l}\text { Abaetetub } \\
\text { a, Brazil }\end{array}$ & $\begin{array}{l}\text { Gois et al., } \\
2016\end{array}$ \\
\hline & Attalea bassleriana (Burret) & Studying, recording and & Fractures & Direct use & Topic & Brazil- & Rengifo- \\
\hline
\end{tabular}


Iriartea deltoidea R\&P. Studying, recording and disseminating ethno-knowledge

\begin{tabular}{|c|c|c|c|c|c|c|c|}
\hline & Iriartea deltoidea R.\&P. & $\begin{array}{c}\text { Studying, recording and } \\
\text { disseminating ethno-knowledge }\end{array}$ & Stomach & Direct use & Oral & $\begin{array}{c}\text { Brazil- } \\
\text { Colombia- } \\
\text { Peru } \\
\text { Border } \\
\end{array}$ & $\begin{array}{l}\text { Rengifo- } \\
\text { Salgado et } \\
\text { al., } 2017\end{array}$ \\
\hline \multirow{5}{*}{ Euphorbiaceae } & Phyllanthus niruri L. & $\begin{array}{c}\text { Studying, recording and } \\
\text { disseminating ethno-knowledge }\end{array}$ & $\begin{array}{l}\text { Fever, kidney and } \\
\text { gallstones }\end{array}$ & Cooked & Oral & $\begin{array}{c}\text { Brazil- } \\
\text { Colombia- } \\
\text { Peru } \\
\text { Border }\end{array}$ & $\begin{array}{l}\text { Rengifo- } \\
\text { Salgado et } \\
\text { al., } 2017\end{array}$ \\
\hline & Croton lechleri Muell Arg. & $\begin{array}{c}\text { Studying, recording and } \\
\text { disseminating ethno-knowledge }\end{array}$ & Healing & Direct use & Topic & $\begin{array}{l}\text { Brazil- } \\
\text { Colombia- } \\
\text { Peru } \\
\text { Border }\end{array}$ & $\begin{array}{l}\text { Rengifo- } \\
\text { Salgado et } \\
\text { al., } 2017\end{array}$ \\
\hline & Jatropha curcas L. & $\begin{array}{c}\text { Studying, recording and } \\
\text { disseminating ethno-knowledge }\end{array}$ & $\begin{array}{l}\text { Alcoholism, fever, } \\
\text { gastritis and wounds }\end{array}$ & Direct use & Oral and topic & $\begin{array}{l}\text { Brazil- } \\
\text { Colombia- } \\
\text { Peru } \\
\text { Border }\end{array}$ & $\begin{array}{l}\text { Rengifo- } \\
\text { Salgado et } \\
\text { al., } 2017\end{array}$ \\
\hline & Jatropha gossypiifolia L. & $\begin{array}{l}\text { Rescuing traditional knowledge } \\
\text { about medicinal plants }\end{array}$ & $\begin{array}{l}\text { "Athlete's foot" and } \\
\text { cold }\end{array}$ & $\begin{array}{l}\text { Decoction and } \\
\text { bath }\end{array}$ & $\begin{array}{l}\text { Internal and } \\
\text { external use }\end{array}$ & $\begin{array}{l}\text { Rondônia, } \\
\text { Brazil }\end{array}$ & $\begin{array}{l}\text { Santos and } \\
\text { Lima } 2014\end{array}$ \\
\hline & Hura crepitans L. & $\begin{array}{c}\text { Collect information on plant } \\
\text { ethnomedicine in general }\end{array}$ & $\begin{array}{c}\text { Snake bite and } \\
\text { injuries }\end{array}$ & $\begin{array}{l}\text { Decoction, dye, } \\
\text { fresh and infusion }\end{array}$ & $\begin{array}{l}\text { Internal and } \\
\text { external use }\end{array}$ & $\begin{array}{l}\text { Paranapur } \\
\text { a, Peru }\end{array}$ & $\begin{array}{c}\text { Odonne et } \\
\text { al., } 2013\end{array}$ \\
\hline \multirow{3}{*}{ Moraceae } & Ficus insipida Wildenow & $\begin{array}{c}\text { Studying, recording and } \\
\text { disseminating ethno-knowledge }\end{array}$ & Hernias & Direct use & Topic & $\begin{array}{c}\text { Brazil- } \\
\text { Colombia- } \\
\text { Peru } \\
\text { Border }\end{array}$ & $\begin{array}{l}\text { Rengifo- } \\
\text { Salgado et } \\
\text { al., } 2017\end{array}$ \\
\hline & $\begin{array}{c}\text { Brosimum guianense (Aubl) } \\
\text { Huber }\end{array}$ & $\begin{array}{c}\text { Studying, recording and } \\
\text { disseminating ethno-knowledge }\end{array}$ & "Womb" cancer & Cooked & Oral & $\begin{array}{l}\text { Brazil- } \\
\text { Colombia- } \\
\text { Peru } \\
\text { Border }\end{array}$ & $\begin{array}{l}\text { Rengifo- } \\
\text { Salgado et } \\
\text { al., } 2017\end{array}$ \\
\hline & Ficus guianensis Desvaus & $\begin{array}{c}\text { Studying, recording and } \\
\text { disseminating ethno-knowledge }\end{array}$ & $\begin{array}{l}\text { Rheumatism and } \\
\text { fractures }\end{array}$ & Direct use & Topic & $\begin{array}{c}\text { Brazil- } \\
\text { Colombia- } \\
\text { Peru } \\
\text { Border } \\
\end{array}$ & $\begin{array}{l}\text { Rengifo- } \\
\text { Salgado et } \\
\text { al., } 2017\end{array}$ \\
\hline Rubiaceae & Genipa americana $\mathrm{L}$. & $\begin{array}{c}\text { Studying, recording and } \\
\text { disseminating ethno-knowledge }\end{array}$ & Antiparasitic & Infusion & Oral & $\begin{array}{l}\text { Brazil- } \\
\text { Colombia- } \\
\text { Peru }\end{array}$ & $\begin{array}{l}\text { Rengifo- } \\
\text { Salgado et } \\
\text { al., } 2017\end{array}$ \\
\hline
\end{tabular}

Border

Brazil- 


\begin{tabular}{|c|c|c|c|c|c|c|c|}
\hline & & & & & & Border & \\
\hline & $\begin{array}{l}\text { Faramea anisocalyx } \\
\text { Poeppig }\end{array}$ & $\begin{array}{l}\text { Collect information on plant } \\
\text { ethnomedicine in general }\end{array}$ & Anemia & Decoction & Shower & $\begin{array}{l}\text { Paranapur } \\
\text { a, Peru }\end{array}$ & $\begin{array}{l}\text { Odonne et } \\
\text { al., } 2013\end{array}$ \\
\hline & $\begin{array}{l}\text { Uncaria guianensis (Aublet) } \\
\text { Gmelin }\end{array}$ & $\begin{array}{c}\text { Studying, recording and } \\
\text { disseminating ethno-knowledge }\end{array}$ & $\begin{array}{l}\text { Gastritis, cancer, } \\
\text { prostate, liver and } \\
\text { pain }\end{array}$ & Cooked & Oral & $\begin{array}{l}\text { Brazil- } \\
\text { Colombia- } \\
\text { Peru } \\
\text { Border }\end{array}$ & $\begin{array}{l}\text { Rengifo- } \\
\text { Salgado et } \\
\text { al., } 2017\end{array}$ \\
\hline & Uncaria tomentosa D.C. & $\begin{array}{l}\text { Recognize the plants used to treat } \\
\text { women's illnesses }\end{array}$ & Contraceptive & Decoction & Intern use & $\begin{array}{l}\text { Leticia, } \\
\text { Colombia } \\
\text { and Puerto } \\
\text { Nariño, } \\
\text { Brazil }\end{array}$ & $\begin{array}{l}\text { Castillo } \\
2015\end{array}$ \\
\hline & $\begin{array}{l}\text { Calycophyllum spruceanum } \\
\text { (Benth.) K.Schum. }\end{array}$ & $\begin{array}{l}\text { Recognize the plants used to treat } \\
\text { women's illnesses }\end{array}$ & Vaginal pains & Decoction & Oral & $\begin{array}{l}\text { Leticia, } \\
\text { Colombia } \\
\text { and Puerto } \\
\text { Nariño, } \\
\text { Brazil } \\
\end{array}$ & $\begin{array}{l}\text { Castillo } \\
2015\end{array}$ \\
\hline Annonaceae & Unonopsis spectabilis Diels & $\begin{array}{c}\text { Studying, recording and } \\
\text { disseminating ethno-knowledge }\end{array}$ & Gastritis & Infusion & Oral & $\begin{array}{l}\text { Brazil- } \\
\text { Colombia- } \\
\text { Peru } \\
\text { Border } \\
\end{array}$ & $\begin{array}{l}\text { Rengifo- } \\
\text { Salgado et } \\
\text { al., } 2017\end{array}$ \\
\hline Rhamnaceae & $\begin{array}{c}\text { Ampelozizyphus amazonicus } \\
\text { Ducke }\end{array}$ & $\begin{array}{c}\text { Investigate, rescue and report } \\
\text { popular knowledge about medicinal } \\
\text { plants }\end{array}$ & $\begin{array}{l}\text { Malaria, liver and } \\
\text { "strengthening the } \\
\text { blood" }\end{array}$ & Maceration & Oral & $\begin{array}{l}\text { Manaus, } \\
\text { Brazil }\end{array}$ & $\begin{array}{l}\text { Veiga and } \\
\text { Scudeller } \\
2015 \\
\end{array}$ \\
\hline \multirow[b]{3}{*}{ Apocynaceae } & $\begin{array}{c}\text { Aspidosperma rigidum } \\
\text { Rusby }\end{array}$ & $\begin{array}{l}\text { Select plants used to treat malaria } \\
\text { and fever }\end{array}$ & $\begin{array}{l}\text { Malaria, liver, fever } \\
\text { and migraine }\end{array}$ & ----- & ----- & $\begin{array}{l}\text { Oriximina } \\
\text {, Brazil }\end{array}$ & $\begin{array}{l}\text { Oliveira et } \\
\text { al., } 2015\end{array}$ \\
\hline & $\begin{array}{c}\text { Geissospermum argenteum } \\
\text { Woodson }\end{array}$ & $\begin{array}{l}\text { Select plants used to treat malaria } \\
\text { and fever }\end{array}$ & $\begin{array}{l}\text { Malaria, liver and } \\
\text { hepatitis }\end{array}$ & ------ & & $\begin{array}{l}\text { Oriximina } \\
\text {, Brazil }\end{array}$ & $\begin{array}{l}\text { Oliveira et } \\
\text { al., } 2015\end{array}$ \\
\hline & $\begin{array}{l}\text { Himatanthus sucuuba (Spr. } \\
\text { Ex Müll.Arg.) W. }\end{array}$ & $\begin{array}{l}\text { Select plants used to treat malaria } \\
\text { and fever; select species for the } \\
\text { treatment of gastrointestinal } \\
\text { disorders }\end{array}$ & Malaria and gastritis & -------- & --------- & $\begin{array}{l}\text { Oriximina } \\
\text {, Brazil } \\
\text { and } \\
\text { Abaetetub } \\
\text { a, Brazil }\end{array}$ & $\begin{array}{l}\text { Oliveira et } \\
\text { al., 2015; } \\
\text { Gois et al., } \\
\quad 2016\end{array}$ \\
\hline \multirow[t]{2}{*}{ Solanaceae } & Physalis angulata $\mathrm{L}$. & $\begin{array}{l}\text { Document indigenous knowledge } \\
\text { about the use of medicinal plants }\end{array}$ & $\begin{array}{l}\text { Fungi on the skin and } \\
\text { cholelithiasis }\end{array}$ & $\begin{array}{l}\text { Juice, infusion } \\
\text { and decoction }\end{array}$ & $\begin{array}{l}\text { Internal and } \\
\text { external use }\end{array}$ & $\begin{array}{l}\text { Coronel } \\
\text { Portillo, } \\
\text { Peru }\end{array}$ & $\begin{array}{l}\text { Polesna et } \\
\text { al., } 2011\end{array}$ \\
\hline & Solanum mammosum $\mathrm{L}$. & Describe traditional medicine and & Skin fungi, infertility & Decoction and & Internal use & Porvenir, & Hajdu and \\
\hline
\end{tabular}




\begin{tabular}{|c|c|c|c|c|c|c|c|}
\hline & & $\begin{array}{l}\text { analyze plants that can be } \\
\text { investigated pharmacologically }\end{array}$ & and skin infections & poultice & & Bolivia & $\begin{array}{l}\text { Hohmann } \\
2012\end{array}$ \\
\hline & Solanum sessiliflorum Dunal & $\begin{array}{l}\text { Recognize the plants used to treat } \\
\text { women's illnesses }\end{array}$ & Burns and diabetes & $\begin{array}{l}\text { Juice, tincture and } \\
\text { decoction }\end{array}$ & $\begin{array}{l}\text { Internal and } \\
\text { external use }\end{array}$ & $\begin{array}{c}\text { Leticia, } \\
\text { Colombia } \\
\text { and Puerto } \\
\text { Nariño, } \\
\text { Brazil }\end{array}$ & $\begin{array}{l}\text { Castillo } \\
2015\end{array}$ \\
\hline \multirow{5}{*}{ Asteraceae } & Mikania lindleyana DC. & $\begin{array}{l}\text { Select species for the treatment of } \\
\text { gastrointestinal disorders }\end{array}$ & Stomachache & --------- & --------- & $\begin{array}{l}\text { Abaetetub } \\
\text { a, Brazil }\end{array}$ & $\begin{array}{l}\text { Gois et al., } \\
2016\end{array}$ \\
\hline & $\begin{array}{l}\text { Pluchea sagittalis (Lam.) } \\
\text { Cabrera }\end{array}$ & $\begin{array}{l}\text { Select species for the treatment of } \\
\text { gastrointestinal disorders }\end{array}$ & $\begin{array}{l}\text { Stomach pain and } \\
\text { gastritis }\end{array}$ & ---- & & $\begin{array}{l}\text { Abaetetub } \\
\text { a, Brazil }\end{array}$ & $\begin{array}{l}\text { Gois et al., } \\
2016\end{array}$ \\
\hline & Vernonia condensata Baker. & $\begin{array}{l}\text { Select species for the treatment of } \\
\text { gastrointestinal disorders }\end{array}$ & $\begin{array}{c}\text { Stomach pain and } \\
\text { diarrhea }\end{array}$ & ------ & 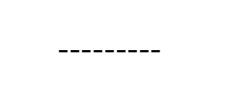 & $\begin{array}{l}\text { Abaetetub } \\
\text { a, Brazil }\end{array}$ & $\begin{array}{l}\text { Gois et al., } \\
2016\end{array}$ \\
\hline & $\begin{array}{l}\text { Spilanthes acmella (L.) } \\
\text { Murray }\end{array}$ & $\begin{array}{c}\text { Investigate, rescue and report } \\
\text { popular knowledge about medicinal } \\
\text { plants }\end{array}$ & Liver & --------- & --------- & $\begin{array}{l}\text { Manaus, } \\
\text { Brazil }\end{array}$ & $\begin{array}{l}\text { Veiga and } \\
\text { Scudeller } \\
2015\end{array}$ \\
\hline & $\begin{array}{c}\text { Achyrocline satureioides } \\
\text { D.C. }\end{array}$ & $\begin{array}{c}\text { Investigate, rescue and report } \\
\text { popular knowledge about medicinal } \\
\text { plants }\end{array}$ & $\begin{array}{l}\text { Stomach, fever and } \\
\text { malaria }\end{array}$ & -------- & --------- & $\begin{array}{l}\text { Manaus, } \\
\text { Brazil }\end{array}$ & $\begin{array}{l}\text { Veiga and } \\
\text { Scudeller } \\
2015 \\
\end{array}$ \\
\hline \multirow{3}{*}{ Lamiaceae } & Mentha sp. & $\begin{array}{l}\text { Select species for the treatment of } \\
\text { gastrointestinal disorders }\end{array}$ & $\begin{array}{l}\text { Stomach and "belly" } \\
\text { pain }\end{array}$ & ------- & & $\begin{array}{l}\text { Abaetetub } \\
\text { a, Brazil }\end{array}$ & $\begin{array}{l}\text { Gois et al., } \\
2016\end{array}$ \\
\hline & $\begin{array}{l}\text { Hyptis crenata Pohl ex. } \\
\text { Benth }\end{array}$ & $\begin{array}{l}\text { Select species for the treatment of } \\
\text { gastrointestinal disorders }\end{array}$ & $\begin{array}{l}\text { Bellyache, diarrhea } \\
\text { and colic }\end{array}$ & ------. & & $\begin{array}{l}\text { Abaetetub } \\
\text { a, Brazil }\end{array}$ & $\begin{array}{l}\text { Gois et al., } \\
2016\end{array}$ \\
\hline & Coleus sp. & $\begin{array}{l}\text { Select species for the treatment of } \\
\text { gastrointestinal disorders }\end{array}$ & Stomachache & --------- & --------- & $\begin{array}{l}\text { Abaetetub } \\
\text { a, Brazil }\end{array}$ & $\begin{array}{l}\text { Gois et al., } \\
2016\end{array}$ \\
\hline \multirow{3}{*}{ Anacardiaceae } & $\begin{array}{c}\text { Anacardium occidentale } \\
L .\end{array}$ & $\begin{array}{l}\text { Select species for the treatment of } \\
\text { gastrointestinal disorders }\end{array}$ & $\begin{array}{l}\text { Diabetes, ringworm, } \\
\text { diarrhea, gastritis and } \\
\text { inflammation }\end{array}$ & $\begin{array}{l}\text { Cooked } \\
\text { Juice }\end{array}$ & Oral and topic & $\begin{array}{l}\text { Abaetetub } \\
\text { a, Brazil }\end{array}$ & $\begin{array}{l}\text { Gois et al., } \\
2016\end{array}$ \\
\hline & Mangifera indica $L$. & $\begin{array}{l}\text { Check plants used by traditional } \\
\text { medicine }\end{array}$ & $\begin{array}{l}\text { Wound cleaning and } \\
\text { diarrhea }\end{array}$ & Juice & Topic & $\begin{array}{l}\text { Ilha do } \\
\text { Mosqueir } \\
\text { o, Brazil }\end{array}$ & $\begin{array}{l}\text { Mesquita } \\
\text { and } \\
\text { Tavares- } \\
\text { Mastins } \\
2018\end{array}$ \\
\hline & $\begin{array}{c}\text { Anacardium humile A.St.- } \\
\text { Hil. }\end{array}$ & $\begin{array}{l}\text { Check plants used by traditional } \\
\text { medicine }\end{array}$ & Inflammation & Cooked & Oral & $\begin{array}{l}\text { Ilha do } \\
\text { Mosqueir } \\
\text { o, Brazil }\end{array}$ & $\begin{array}{l}\text { Mesquita } \\
\quad \text { and } \\
\text { Tavares- } \\
\text { Mastins }\end{array}$ \\
\hline
\end{tabular}




\begin{tabular}{|c|c|c|c|c|c|c|c|}
\hline & & & & & & & 2018 \\
\hline \multirow{4}{*}{ Piperaceae } & $\begin{array}{l}\text { Peperomia pellucida (L.) } \\
\text { Kunth }\end{array}$ & $\begin{array}{l}\text { Check plants used by traditional } \\
\text { medicine }\end{array}$ & "Earthworms" & Cooked & Oral & $\begin{array}{l}\text { Ilha do } \\
\text { Mosqueir } \\
\text { o, Brazil }\end{array}$ & $\begin{array}{c}\text { Mesquita } \\
\text { and } \\
\text { Tavares- } \\
\text { Mastins } \\
2018\end{array}$ \\
\hline & $\begin{array}{c}\text { Piper alatipetiolatum } \\
\text { Yunck. }\end{array}$ & $\begin{array}{l}\text { Check plants used by traditional } \\
\text { medicine }\end{array}$ & The flu & $\begin{array}{l}\text { Juice and } \\
\text { maceration }\end{array}$ & Topic & $\begin{array}{l}\text { Ilha do } \\
\text { Mosqueir } \\
\text { o, Brazil }\end{array}$ & $\begin{array}{l}\text { Mesquita } \\
\text { and } \\
\text { Tavares- } \\
\text { Mastins } \\
2018\end{array}$ \\
\hline & Piper peltatum L. & $\begin{array}{l}\text { Rescuing traditional knowledge } \\
\text { about medicinal plants }\end{array}$ & $\begin{array}{l}\text { Hepatitis, anemia and } \\
\text { kidney infection }\end{array}$ & $\begin{array}{l}\text { Infusion, } \\
\text { decoction }\end{array}$ & -------- & $\begin{array}{l}\text { Rondônia, } \\
\text { Brazil }\end{array}$ & $\begin{array}{l}\text { Santos and } \\
\text { Lima } 2014\end{array}$ \\
\hline & Pothomorphe umbellata (L.) & $\begin{array}{l}\text { Rescuing traditional knowledge } \\
\text { about medicinal plants }\end{array}$ & $\begin{array}{c}\text { Hepatitis, malaria and } \\
\text { headache }\end{array}$ & Maceration & --------- & $\begin{array}{c}\text { Rondônia, } \\
\text { Brazil }\end{array}$ & $\begin{array}{l}\text { Santos and } \\
\text { Lima } 2014\end{array}$ \\
\hline \multirow{4}{*}{ Rutaceae } & Ruta graveolens $L$. & $\begin{array}{l}\text { Check plants used by traditional } \\
\text { medicine }\end{array}$ & Headache & Maceration & Topic & $\begin{array}{l}\text { Ilha do } \\
\text { Mosqueir } \\
\text { o, Brazil }\end{array}$ & $\begin{array}{c}\text { Mesquita } \\
\text { and } \\
\text { Tavares- } \\
\text { Mastins } \\
2018\end{array}$ \\
\hline & Citrus reticulata & $\begin{array}{c}\text { Describe traditional medicine and } \\
\text { analyze plants that can be } \\
\text { investigated pharmacologically }\end{array}$ & $\begin{array}{l}\text { Eyes and stomach } \\
\text { pain }\end{array}$ & Infusion & Intern use & $\begin{array}{l}\text { Porvenir, } \\
\text { Bolivia }\end{array}$ & $\begin{array}{l}\text { Hajdu and } \\
\text { Hohmann } \\
2012\end{array}$ \\
\hline & Citrus sinensis (L.) Osbeck & $\begin{array}{c}\text { Investigate, rescue and report } \\
\text { popular knowledge about medicinal } \\
\text { plants }\end{array}$ & Stomach and liver & --------- & ------ & $\begin{array}{l}\text { Manaus, } \\
\text { Brazil }\end{array}$ & $\begin{array}{l}\text { Veiga and } \\
\text { Scudeller } \\
2015\end{array}$ \\
\hline & Citrus cf. aurantium L. & $\begin{array}{c}\text { Investigate, rescue and report } \\
\text { popular knowledge about medicinal } \\
\text { plants }\end{array}$ & Anemia & -------- & --------- & $\begin{array}{l}\text { Manaus, } \\
\text { Brazil }\end{array}$ & $\begin{array}{c}\text { Veiga and } \\
\text { Scudeller } \\
2015 \\
\end{array}$ \\
\hline \multirow{2}{*}{ Myrtaceae } & Eucalyptus sp. & $\begin{array}{c}\text { Investigate, rescue and report } \\
\text { popular knowledge about medicinal } \\
\text { plants }\end{array}$ & Fever & --------- & ------- & $\begin{array}{l}\text { Manaus, } \\
\text { Brazil }\end{array}$ & $\begin{array}{l}\text { Veiga and } \\
\text { Scudeller } \\
2015\end{array}$ \\
\hline & Eugenia malaccensis L. & $\begin{array}{c}\text { Investigate, rescue and report } \\
\text { popular knowledge about medicinal } \\
\text { plants }\end{array}$ & Anemia & --------- & --------- & $\begin{array}{l}\text { Manaus, } \\
\text { Brazil }\end{array}$ & $\begin{array}{c}\text { Veiga and } \\
\text { Scudeller } \\
2015 \\
\end{array}$ \\
\hline Zingiberaceae & Zingiber officinale Roscoe & $\begin{array}{l}\text { Check plants used by traditional } \\
\text { medicine }\end{array}$ & Cough & Syrup & Oral & $\begin{array}{l}\text { Ilha do } \\
\text { Mosqueir } \\
\text { o, Brazil }\end{array}$ & $\begin{array}{l}\text { Mesquita } \\
\text { and } \\
\text { Tavares- }\end{array}$ \\
\hline
\end{tabular}




\begin{tabular}{|c|c|c|c|c|c|c|c|}
\hline & $\begin{array}{c}\text { Alpinia speciosa (Blume) D. } \\
\text { Dietr. }\end{array}$ & $\begin{array}{c}\text { Investigate, rescue and report } \\
\text { popular knowledge about medicinal } \\
\text { plants }\end{array}$ & Fever & --------- & --------- & $\begin{array}{l}\text { Manaus, } \\
\text { Brazil }\end{array}$ & $\begin{array}{c}\text { Mastins } \\
2018 \\
\text { Veiga and } \\
\text { Scudeller } \\
2015 \\
\end{array}$ \\
\hline \multirow{2}{*}{ Alligatoridae } & Caiman crocodilus & $\begin{array}{l}\text { Conduct research with traditional } \\
\text { healing experts }\end{array}$ & Psychoactive & --------- & - ------ & $\begin{array}{l}\text { Barcelos, } \\
\text { Brazil }\end{array}$ & $\begin{array}{l}\text { Santos et } \\
\text { al., } 2012\end{array}$ \\
\hline & Paleosuchus trigonatus & $\begin{array}{l}\text { Conduct research with traditional } \\
\text { healing experts }\end{array}$ & Psychoactive & -------- & --------- & $\begin{array}{c}\text { Barcelos, } \\
\text { Brazil }\end{array}$ & $\begin{array}{l}\text { Santos et } \\
\text { al., } 2012\end{array}$ \\
\hline \multirow[t]{2}{*}{ Humiriaceae } & $\begin{array}{c}\text { Endopleura uchi (Huber) } \\
\text { Cuatrec }\end{array}$ & $\begin{array}{l}\text { Check plants used by traditional } \\
\text { medicine }\end{array}$ & $\begin{array}{l}\text { Inflammation and } \\
\text { diabetes }\end{array}$ & Cooked & Oral & $\begin{array}{l}\text { Ilha do } \\
\text { Mosqueir } \\
\text { o, Brazil }\end{array}$ & $\begin{array}{c}\text { Mesquita } \\
\text { and } \\
\text { Tavares- } \\
\text { Mastins } \\
2018\end{array}$ \\
\hline & $\begin{array}{l}\text { Schistostemon } \\
\text { macrophyllum }\end{array}$ & $\begin{array}{l}\text { Conduct research with traditional } \\
\text { healing experts }\end{array}$ & Contraceptive & --------- & --------- & $\begin{array}{l}\text { Barcelos, } \\
\text { Brazil }\end{array}$ & $\begin{array}{l}\text { Santos et } \\
\text { al., } 2012\end{array}$ \\
\hline \multirow{3}{*}{ Burseraceae } & $\begin{array}{l}\text { Protium amazonicum } \\
\text { (Cuatrec.) }\end{array}$ & $\begin{array}{l}\text { Conduct research with traditional } \\
\text { healing experts }\end{array}$ & Analgesic & --------- & --------- & $\begin{array}{l}\text { Barcelos, } \\
\text { Brazil }\end{array}$ & $\begin{array}{l}\text { Santos et } \\
\text { al., } 2012\end{array}$ \\
\hline & $\begin{array}{l}\text { Protium cf. aracouchini } \\
\text { (Aubl.) }\end{array}$ & $\begin{array}{l}\text { Conduct research with traditional } \\
\text { healing experts }\end{array}$ & Analgesic & --------- & --------- & $\begin{array}{l}\text { Barcelos, } \\
\text { Brazil }\end{array}$ & $\begin{array}{l}\text { Santos et } \\
\text { al., } 2012\end{array}$ \\
\hline & $\begin{array}{l}\text { Protium cf. heptaphyllum } \\
\text { (Aubl.) }\end{array}$ & $\begin{array}{l}\text { Conduct research with traditional } \\
\text { healing experts }\end{array}$ & Analgesic & -------- & --------- & $\begin{array}{l}\text { Barcelos, } \\
\text { Brazil }\end{array}$ & $\begin{array}{l}\text { Santos et } \\
\text { al., } 2012\end{array}$ \\
\hline \multirow{4}{*}{ Cichlidae } & Acaronia nassa & $\begin{array}{l}\text { Conduct research with traditional } \\
\text { healing experts }\end{array}$ & Respiratory system & --------- & -------- & $\begin{array}{l}\text { Barcelos, } \\
\text { Brazil }\end{array}$ & $\begin{array}{l}\text { Santos et } \\
\text { al., } 2012\end{array}$ \\
\hline & Crenicichla cincta & $\begin{array}{l}\text { Conduct research with traditional } \\
\text { healing experts }\end{array}$ & Respiratory system & --------- & --------- & $\begin{array}{l}\text { Barcelos, } \\
\text { Brazil }\end{array}$ & $\begin{array}{l}\text { Santos et } \\
\text { al., } 2012\end{array}$ \\
\hline & Mesonauta insignis & $\begin{array}{l}\text { Conduct research with traditional } \\
\text { healing experts }\end{array}$ & Respiratory system & --------- & --------- & $\begin{array}{l}\text { Barcelos, } \\
\text { Brazil }\end{array}$ & $\begin{array}{l}\text { Santos et } \\
\text { al., } 2012\end{array}$ \\
\hline & Satanoperca jurupari & $\begin{array}{l}\text { Conduct research with traditional } \\
\text { healing experts }\end{array}$ & Respiratory system & --------- & --------- & $\begin{array}{c}\text { Barcelos, } \\
\text { Brazil }\end{array}$ & $\begin{array}{l}\text { Santos et } \\
\text { al., } 2012\end{array}$ \\
\hline \multirow[t]{2}{*}{ Poaceae } & Cymbopogon citratus & $\begin{array}{l}\text { Check plants used by traditional } \\
\text { medicine }\end{array}$ & Stomach & $\begin{array}{l}\text { Cooked, } \\
\text { maceration and } \\
\text { infusion }\end{array}$ & Topic and oral & $\begin{array}{l}\text { Ilha do } \\
\text { Mosqueir } \\
\text { o, Brazil }\end{array}$ & $\begin{array}{c}\text { Mesquita } \\
\text { and } \\
\text { Tavares- } \\
\text { Mastins } \\
2018\end{array}$ \\
\hline & Oryza sativa L. & $\begin{array}{l}\text { Describe traditional medicine and } \\
\text { analyze plants that can be }\end{array}$ & Stomach & $\begin{array}{l}\text { Decoction, } \\
\text { toasting and }\end{array}$ & Intern use & $\begin{array}{l}\text { Porvenir, } \\
\text { Bolivia }\end{array}$ & $\begin{array}{l}\text { Hajdu and } \\
\text { Hohmann }\end{array}$ \\
\hline
\end{tabular}




\begin{tabular}{|c|c|c|c|c|c|c|c|}
\hline & Zea mays L. & $\begin{array}{l}\text { investigated pharmacologically } \\
\text { Describe traditional medicine and } \\
\text { analyze plants that can be } \\
\text { investigated pharmacologically }\end{array}$ & Kidneys & grinding & Intern use & $\begin{array}{l}\text { Porvenir, } \\
\text { Bolivia }\end{array}$ & $\begin{array}{l}2012 \\
\text { Hajdu and } \\
\text { Hohmann } \\
2012\end{array}$ \\
\hline Celastraceae & Maytenus krukovii & $\begin{array}{l}\text { Collect information on plant } \\
\text { ethnomedicine in general }\end{array}$ & $\begin{array}{l}\text { Anemia and } \\
\text { rheumatism }\end{array}$ & Decoction & -------- & $\begin{array}{c}\text { Paranapur } \\
\text { a, Peru }\end{array}$ & $\begin{array}{l}\text { Odonne et } \\
\text { al., } 2013\end{array}$ \\
\hline \multirow{2}{*}{$\begin{array}{c}\text { Amaranthacea } \\
\mathrm{e}\end{array}$} & $\begin{array}{l}\text { Chenopodium } \\
\text { ambrosioides }\end{array}$ & $\begin{array}{l}\text { Select species for the treatment of } \\
\text { gastrointestinal disorders; rescue } \\
\text { traditional knowledge about } \\
\text { medicinal plants }\end{array}$ & $\begin{array}{l}\text { Diarrhea, nausea, } \\
\text { stomach pain and } \\
\text { tuberculosis }\end{array}$ & --------- & --------- & $\begin{array}{c}\text { Abaetetub } \\
\text { a, Brazil } \\
\text { and } \\
\text { Rondônia, } \\
\text { Brazil }\end{array}$ & $\begin{array}{l}\text { Gois et al., } \\
2016 ; \\
\text { Santos and } \\
\text { Lima } 2014\end{array}$ \\
\hline & $\begin{array}{c}\text { Alternanthera dentata } \\
\text { (Moench) Stuchlík ex } \\
\text { R.E.Fr. }\end{array}$ & $\begin{array}{l}\text { Rescuing traditional knowledge } \\
\text { about medicinal plants }\end{array}$ & $\begin{array}{l}\text { Infections and } \\
\text { inflammation }\end{array}$ & $\begin{array}{l}\text { Infusion and } \\
\text { decoction }\end{array}$ & --------- & $\begin{array}{l}\text { Rondônia, } \\
\text { Brazil }\end{array}$ & $\begin{array}{l}\text { Santos and } \\
\text { Lima } 2014\end{array}$ \\
\hline \multirow{3}{*}{ Malvaceae } & $\begin{array}{l}\text { Ceiba pentandra }(\mathrm{L} .) \\
\text { Gaertn. }\end{array}$ & $\begin{array}{l}\text { Recognize the plants used to treat } \\
\text { women's illnesses }\end{array}$ & Fertility & Maceration & Bath and oral & $\begin{array}{c}\text { Leticia, } \\
\text { Colombia } \\
\text { and Puerto } \\
\text { Nariño, } \\
\text { Brazil }\end{array}$ & $\begin{array}{l}\text { Castillo } \\
2015\end{array}$ \\
\hline & Gossypium herbaceum L. & $\begin{array}{l}\text { Recognize the plants used to treat } \\
\text { women's illnesses }\end{array}$ & $\begin{array}{l}\text { Fertility and } \\
\text { menstrual cramps }\end{array}$ & Decoction & Oral & $\begin{array}{c}\text { Leticia, } \\
\text { Colombia } \\
\text { and } \\
\text { Puerto } \\
\text { Nariño, } \\
\text { Brazil }\end{array}$ & $\begin{array}{l}\text { Castillo } \\
2015\end{array}$ \\
\hline & Hibiscus sabdariffa $\mathrm{L}$. & $\begin{array}{l}\text { Recognize the plants used to treat } \\
\text { women's illnesses }\end{array}$ & $\begin{array}{l}\text { Regulation of } \\
\text { menopause }\end{array}$ & Infusion & Oral & $\begin{array}{l}\text { Leticia, } \\
\text { Colômbia } \\
\text { and Puerto } \\
\text { Nariño, } \\
\text { Brazil }\end{array}$ & $\begin{array}{l}\text { Castillo } \\
2015\end{array}$ \\
\hline Meliaceae & Carapa guianensis Aubl. & $\begin{array}{l}\text { Conduct research with traditional } \\
\text { healing experts }\end{array}$ & $\begin{array}{l}\text { Anti-inflammatory } \\
\text { and dermatological } \\
\text { and urinary disorders }\end{array}$ & Exudato & --------- & $\begin{array}{l}\text { Barcelos, } \\
\text { Brazil }\end{array}$ & $\begin{array}{l}\text { Santos et } \\
\text { al., } 2012\end{array}$ \\
\hline Sapindaceae & $\begin{array}{c}\text { Melicoccus lepidopetalus } \\
\text { Radlk }\end{array}$ & $\begin{array}{l}\text { Describe traditional medicine and } \\
\text { analyze plants that can be } \\
\text { investigated pharmacologically }\end{array}$ & $\begin{array}{l}\text { Diarrhea and } \\
\text { dysentery }\end{array}$ & Decoction & Intern use & $\begin{array}{l}\text { Porvenir, } \\
\text { Bolivia }\end{array}$ & $\begin{array}{l}\text { Hajdu and } \\
\text { Hohmann } \\
2012\end{array}$ \\
\hline
\end{tabular}




\begin{tabular}{|c|c|c|c|c|c|c|c|}
\hline & Scoparia dulcis L. & $\begin{array}{l}\text { Recognize the plants used to treat } \\
\text { women's illnesses }\end{array}$ & $\begin{array}{l}\text { Hematoma, } \\
\text { inflammation and } \\
\text { toothache }\end{array}$ & Infusion & Intern use & $\begin{array}{c}\text { Leticia, } \\
\text { Colombia } \\
\text { and Puerto } \\
\text { Nariño, } \\
\text { Brazil }\end{array}$ & $\begin{array}{l}\text { Castillo } \\
2015\end{array}$ \\
\hline \multirow{3}{*}{ Cucurbitaceae } & Lagenaria siceraria & $\begin{array}{l}\text { Describe traditional medicine and } \\
\text { analyze plants that can be } \\
\text { investigated pharmacologically }\end{array}$ & Scabies and furuncle & $\begin{array}{l}\text { Grinding and } \\
\text { rough }\end{array}$ & Shower & $\begin{array}{c}\text { Porvenir, } \\
\text { Bolivia }\end{array}$ & $\begin{array}{l}\text { Hajdu and } \\
\text { Hohmann } \\
2012\end{array}$ \\
\hline & Citrullus lanatus & $\begin{array}{l}\text { Describe traditional medicine and } \\
\text { analyze plants that can be } \\
\text { investigated pharmacologically }\end{array}$ & Vomiting & Grinding & Intern use & $\begin{array}{l}\text { Porvenir, } \\
\text { Bolivia }\end{array}$ & $\begin{array}{l}\text { Hajdu and } \\
\text { Hohmann } \\
2012\end{array}$ \\
\hline & Cucurbita sp. & $\begin{array}{l}\text { Describe traditional medicine and } \\
\text { analyze plants that can be } \\
\text { investigated pharmacologically }\end{array}$ & $\begin{array}{l}\text { Rapture and } \\
\text { remastered }\end{array}$ & $\begin{array}{l}\text { Grinding and } \\
\text { decoction }\end{array}$ & Massage & $\begin{array}{l}\text { Porvenir, } \\
\text { Bolivia }\end{array}$ & $\begin{array}{c}\text { Hajdu and } \\
\text { Hohmann } \\
2012 \\
\end{array}$ \\
\hline \multirow{3}{*}{ Bignoniaceae } & Crescentia cujete L. & $\begin{array}{l}\text { Recognize the plants used to treat } \\
\text { women's illnesses }\end{array}$ & Fertility and healing & $\begin{array}{l}\text { Maceration and } \\
\text { crude }\end{array}$ & $\begin{array}{l}\text { Internal and } \\
\text { external use }\end{array}$ & $\begin{array}{c}\text { Leticia, } \\
\text { Colombia } \\
\text { and Puerto } \\
\text { Nariño, } \\
\text { Brazil }\end{array}$ & $\begin{array}{l}\text { Castillo } \\
2015\end{array}$ \\
\hline & Tabebuia impetiginosa & $\begin{array}{l}\text { Describe traditional medicine and } \\
\text { analyze plants that can be } \\
\text { investigated pharmacologically }\end{array}$ & $\begin{array}{l}\text { Rheumatism, malaria, } \\
\text { kidneys and cancer }\end{array}$ & Decoction & Intern use & $\begin{array}{l}\text { Porvenir, } \\
\text { Bolivia }\end{array}$ & $\begin{array}{l}\text { Hajdu and } \\
\text { Hohmann } \\
2012\end{array}$ \\
\hline & Tabebuia aurea & $\begin{array}{l}\text { Describe traditional medicine and } \\
\text { analyze plants that can be } \\
\text { investigated pharmacologically }\end{array}$ & $\begin{array}{c}\text { Appendicitis, fever, } \\
\text { weakness, liver and } \\
\text { kidneys } \\
\end{array}$ & Decoction & Intern use & $\begin{array}{l}\text { Porvenir, } \\
\text { Bolivia }\end{array}$ & $\begin{array}{c}\text { Hajdu and } \\
\text { Hohmann } \\
2012 \\
\end{array}$ \\
\hline \multirow[t]{2}{*}{ Cyperaceae } & Cyperus articulatus L. & $\begin{array}{l}\text { Check plants used by traditional } \\
\text { medicine }\end{array}$ & Asthma & Cooked & Oral & $\begin{array}{l}\text { Ilha do } \\
\text { Mosqueir } \\
\text { o, Brazil }\end{array}$ & $\begin{array}{c}\text { Mesquita } \\
\text { and } \\
\text { Tavares- } \\
\text { Mastins } \\
2018\end{array}$ \\
\hline & Cyperus prolixus Kunth & $\begin{array}{l}\text { Collect information on plant } \\
\text { ethnomedicine in general }\end{array}$ & Diarrhea & $\begin{array}{l}\text { Crushed or } \\
\text { chewed }\end{array}$ & --------- & $\begin{array}{c}\text { Paranapur } \\
\text { a, Peru }\end{array}$ & $\begin{array}{c}\text { Odonne et } \\
\text { al., } 2013\end{array}$ \\
\hline
\end{tabular}




\section{Discussion}

Ethnobotany is considered an important area of science, as it studies the ecological and cultural influences of plants from ancient and modern society, with great repercussions in pharmacology. In this context, ethnobotanical research extracts information about folk knowledge regarding plants so that studies that aim their beneficial use can be carried out. Thus, in addition to valuing folk knowledge, it can develop technologies to take advantage of these sustainable resources (Cragg; Newman, 2013).

One of the most important practices related to ethnobotany is called a blessing, which is a healing alternative practiced by women, usually called folk healers, who aim to heal through the use of plants and religion (Júnior et al., 2013). According to the evolutionary process, it is observed that women have most of the knowledge of the use of medicinal plants and, thus, the responsibility to take care of family health falls on them (Vásquez et al., 2014) Therefore, the main source of medicinal plants are the backyards of the houses, being them cultivated directly from the ground or flower beds, and, in the background, the forest also ends up serving as a means of access (Carniello et al., 2010).

In the Amazon region, which is home to various indigenous tribes, this practice is widely used by shamans, usually in rituals associated with supernatural factors. In addition to that, plants are also used as amulets, to scare the evil eye, during childbirth and religious rituals (Rocha, 2014). In this place, the knowledge related to the use of medicinal plants is orally transmitted from generation to generation (Freitas, 2014), that is, this knowledge is part of the culture of most people.

In this context, there are studies that indicate a national trend of deficit of scientific knowledge on the part of health professionals and the general population regarding Integrative and Complementary Practices, and a large part of pre-existing knowledge is conditioned to knowledge passed on through parental relationships and/or isolated events (Goés; Silva; Castro, 2019).

In this region, the main way of preparing medicinal plants is tea, which can be prepared in four main ways: (1) infusion, which consists of putting the contents of the plant in boiling water for 15-20 minutes, letting it rest for 5-10 minutes and then straining it is more used for the soft parts of plants, such as flowers or leaves; (2) decoction, which consists of placing the material in cold water and cooking it for 5-30 minutes and then straining it, is indicated for the hard parts of the plant, such as stems, roots, and barks; (3) maceration, which consists of placing the contents in cold water for 10-24 hours and then straining it is used for different parts of the plant, such as flowers, leaves, seeds, stems, roots, and barks; (4) herbal tea, which consists of placing the contents in a pan with boiling water for 5 minutes and then straining it, is used for herbs (Who, 2018).

Medicinal plants are often used in powder form, made from the dried plant, since crushing and sifting is necessary. Then, this powder can be used to prepare infusions, decoctions, or oils. Besides, juices are also widely used, needing only to have its herbs crushed and then strained. Syrups are prepared with a mixture of $50 \%$ juice, decocted or macerated, and $50 \%$ honey. As a form of external use, poultice stands out, a form in which fresh or dried herbs are used, which is placed directly on the spot you want it. The bath is also an external use form, because despite being prepared from an infusion, instead of ingesting it, it is used to wash the body (Who, 2018).

Other ways of using medicinal plants involve inhalation, which consists of placing the contents in boiling water and slowly inhaling the released steam, and tincture intake, prepared as if it were a maceration, but using alcohol, preferably cereals, being consumed in drops diluted in cold water, ointments, or rubs. The tincture form is the one that most preserves the active principles of medicinal plants, since most are soluble in alcohol (Who, 2018).

Even with people's access to traditional medicine, medicinal plants are still widely used for maintaining health in some communities in the Amazon (Vásquez et al., 2014). In this study, the main botanical families used in this region were analyzed with Fabaceae being the most frequent, presenting species pointed out by the population as effective in the treatment 
of gastrointestinal alterations, it is usually used in the form of infusing or cooked (in the colloquial language) for cases of diarrhea and abdominal pain. In addition, they are species used to treat infections, inflammations, liver disorders, and even cancer (Frausin et al., 2015; Gois et al., 2016; Rengifo-Salgado et al., 2017) (Table 1, columns 1, 2, and 4).

The Euphorbiaceae and Rubiaceae families described in different studies are identified by the population as useful for liver, gallbladder, stomach, and kidneys' alterations relief, in addition to pain and fever reducing. These species are mainly used in the forms of decoction, macerated or infused (Odonne et al., 2013; Santos et al., 2014; Castillo, 2015; Giovannini, 2015) (Table 1, columns 1, 2, and 4).

Species of the Solanaceae Family are listed by the population as effective for treating rheumatic alterations and reducing fungal infections. The main ways of preparing and using these species are tea, juice, poultice, and tincture (Polesna et al., 2011; Castillo, 2015; Gois et al., 2016) (Table 1; columns 1, 2, and 4).

Different species belonging to the Asteraceae, Annonaceae, Poaceae and Burseraceae botanical families have been reported to have analgesic potential, to reduce stomach pain and improve gastritis, being prepared in various forms, such as infusion or decoction (Veiga et al., 2015; Gois et al., 2016; Rengifo-Salgado et al., 2017; Mesquita et al., 2018) (Table 1; columns 1, 2, and 4).

The Rhamnaceae and Apocynaceae families present botanical species used by the population for the treatment of malaria, that represents an endemic disease for the Amazon region which can be explored by parasitic research groups, so that better therapies against it can be developed (Oliveira et al., 2015; Veiga et al., 2015; Gois et al., 2016) (Table 1; columns 1, 2, and 4).

Other botanical families were listed by the Amazon region population and were used for intestinal and respiratory disorders, anemia, headaches, and fever, among others (Table1). This knowledge, full of inaccuracies, especially with regard to the method of preparation, indications and scope of the use of plants and herbal medicines, can lead to treatment failure, leading to a future disbelief in the use of this type of treatment by the patient. (Santos; Léda; Oliveira, 2018).

Folk knowledge about medicinal plants has awakened governmental interest, a fact that has supported policies that aim to highlight the importance of alternative medicine for more effective and humanizing health care (Brazil, 2000). Furthermore, there is still a lack of scientific studies that prove the pharmacological properties of the various medicinal plants existing in Brazil, in order to inform the correct dose to be used, the proper method of preparation, the maximum time that it can be stored, the indications and possible drug interactions that may exist with both pharmacy drugs and other medicinal plants

In this sense, Práticas Interativas e Complementares em Saúde (PICS) [Integrative and Complementary Health Practices] were implemented to integrate different medical practices, such as traditional Chinese medicine, homeopathy, and herbal medicine, among others (Telesi-Júnior, 2016). PICS aim to expand therapeutic options to users of the Sistema Único de Saúde (SUS) [Unified Health System], while one of its strategies is to provide access to medicinal plants safely and efficiently (Rodrigues et al., 2006) which was further reinforced by the Programa Nacional de Plantas Medicinais e Fitoterápicos [National Program for Medicinal Plants and Herbal Medicines].

The demand for PICS has increased due to its power to guide the doctor-patient relationship as an important element in therapy, it values simple therapeutic forms, it is less dependent on technologies, and increases the patient's autonomy over the healing process itself (Levin et al., 2001; Nogales-Gaete, 2004; Santos et al., 2012).

Folk medicine study, especially of herbal medicines, has received greater attention from pharmacological research, as they have provided science with a greater amount of information regarding active principles (Vale, 2002). In this sense, folk 
knowledge provides the basis for advances in the fields of therapeutics, emphasizing the importance of updates on traditional medicine.

The limitations of the study involve the difficulty of obtaining more complete information about the plant species used in the Amazon region in published articles, in the sense of correlation with therapeutic use, the chosen plant parts, forms of preparation, route of administration, among others.

\section{Conclusion}

Traditional medicine, practiced through the use of medicinal plants, proves to be of great importance for the Amazon region population, so much so that it is a factor present in the culture of these people and that is passed on from generation to generation. This proves how important this practice is for complementing official medicine. In this sense, it is concluded that the practice of traditional medicine is very common in the Amazon region and is transmitted orally from generation to generation. In this practice, the use of medicinal plants in the form of tea is highlighted, with the species in the form of powder, dried or natural plants. Thus, this study serves as a basis for more in-depth pharmacological investigations to be developed based on the information cited. Therefore, it is suggested that the strengthening of pharmacological studies involving local medicinal plants can provide scientific support for popular knowledge.

\section{References}

Badke, M. R., Budó, M. D., Silva, F. M. \& Ressel, L. B. (2011). Plantas medicinais: o saber sustentado na prática do cotidiano popular. Escola Anna Nery. 15, 132-139.

Braga, P. I. (1979). Subdivisão fitogeográfica, tipos de vegetação, conservação e inventário florístico da floresta amazônica. Acta Amazonica. 9, 53-80.

Brasil (2012). Ministério da Saúde. Caderno de Atenção Básica, n. 31. Práticas integrativas e complementares: plantas medicinais e fitoterapia na Atenção Básica. Brasília, DF.

Brasil (2020). Ministério da Saúde. Agência Nacional de Vigilância Sanitária. Resolução RDC no 17 de 24 de fevereiro de 2000. Aprova o Regulamento técnico visando normatizar o registro de medicamentos fitoterápicos junto ao sistema de vigilância sanitária. Diário Oficial da União 25 Fev $2000 ;$ Seção 1: 25 .

Carniello, M. A., Silva, R. D., Cruz, M. A. \& Guarim-Neto, G. (2010). Quintais urbanos de Mirassol D'Oeste-MT, Brasil: uma abordagem etnobotânica. Acta Amazonica. 40, 451-70.

Castillo, C. (2015). Plantas Medicinales Utilizadas En El Tratamiento De Enfermedades Ginecológicas En Leticia Y Puerto Nariño (Amazonas, Colombia). Etnobiologia.

Cragg, G. M., Newman, D.J. (2013). Natural products: A continuing source of novel drug leads. Biochimica et Biophysica Acta, 1830, $3670-3695$.

Frausin, G., Hidalgo, A. F., Lima, R. B. S., Kinupp, V. F., Ming, L. C., Pohlit, A. M. \& Milliken, W. (2015). An ethnobotanical study of anti-malarial plants among indigenous people on the upper Negro River in the Brazilian Amazon. Journal of Ethnopharmacology. 174, $238-252$.

Freitas, C. L. (2014). Uso terapêutico e religioso das ervas. Revista Caminhos-Revista de Ciências da Religião. 12, 79-92.

Giovannini, P. (2015). Medicinal plants of the Achuar (Jivaro) of Amazonian Ecuador: ethnobotanical survey and comparison with other Amazonian pharmacopoeias. Journal of Ethnopharmacology. 164, 78-88.

Goés, A. C. C., Silva, L. S. L., Castro, N. J. C. (2019). Uso de Plantas Medicinais e Fitoterápacos. Revista de Atenção à Saúde. $17,53-61$.

Gois, M. A. F., Lucas, F. C. A., Costa, J. C. M., Moura, P. H. B. \& Lobato, G. J. M. (2016). Etnobotânica de espécies vegetais medicinais no tratamento de transtornos do sistema gastrointestinal. Revista Brasileira de Plantas Medicinais. 18, 547-557.

Hajdu, Z. \& Hohmann, J. (2012). An ethnopharmacological survey of the traditional medicine utilized in the community of Porvenir, Bajo Paraguá Indian Reservation, Bolivia. Journal of Ethnopharmacology. 139, 838-57.

Júnior, H. R. \& Neves, S. S. (2013). As práticas de benzimento em Parintins: uma abordagem folkcomunicacional. Revista Eletrônica Mutações. 4, 1-12.

Levin, J. S. \& Jonas, W. B. (2001). Tratado de medicina complementar e alternativa. Manole, 1-120.

Mesquita, U. O. \& Tavares-Martins, A. C. (2018). Etnobotánica de plantas medicinales en la comunidad de Caruarú, Isla del Mosqueiro, Belém-PA, Brasil. Boletín Latinoamericano y del Caribe de Plantas Medicinales y aromáticas. 17, 130-159. 
Moher, D., Liberati, A., Tetzlaff, J. \& Altman, D. G. (2009). Preferred Reporting Items for Systematic Reviews and Meta-Analyses: The PRISMA Statement. PLOS Medicine. 6, e1000097.

Nogales-Gaete, J. (2004). Medicina alternativa y complementaria. Revista Chilena de Neuropsiquiatria. 42, $243-250$.

Odonne, G., Valadeau, C., Alban-Castillo, J., Stien, D., Sauvain, M. \& Bourdy, G. (2013). Medical ethnobotany of the Chayahuita of the Paranapura basin (Peruvian Amazon). Journal of Ethnopharmacology. 146, 127-153.

Oliveira, D. R., Krettli, A. U., Aguiar, A. C., Leitão, G. G., Vieira, M. N., Martins, K. S., \& Leitão, S. G. (2015). Ethnopharmacological evaluation of medicinal plants used against malaria by quilombola communities from Oriximiná, Brazil. Journal of Ethnopharmacology. 173, 424-434.

Penna Filho, P. (2013). Reflexões sobre o Brasil e os desafios Pan-Amazônicos. Revista Brasileira de Política Internacional. 56, 94-111.

Polesna, L., Polesny, Z., Clavo, M. Z., Hansson, A. \& Kokoska, L. (2011). Ethnopharmacological inventory of plants used in Coronel Portillo Province of Ucayali Department, Peru. Pharmaceutical Biology. 49, 125-136.

Rengifo-Salgado, E., Rios-Torres, S., Fachín, M. L. \& Vargas-Arana, G. (2017). Saberes ancestrales sobre el uso de flora y fauna en la comunidad indígena Tikuna de Cushillo Cocha, zona fronteriza Perú-Colombia-Brasil. Revista Peruana de Biologia. 24, 67-78.

Rocha, T. T. (2014). Levantamento etnobotânico de plantas utilizadas pelas comunidades da Reserva Extrativista Marinha de Soure, Pará, Brasil. Doctoral dissertation, Programa de Pós-graduação em Ciências Ambientais, UEPA, Belém.

Rodrigues, A. G., Santos, M. G. \& Amaral, A. C. F. (2006). Políticas públicas em plantas medicinais e fitoterápicos. Ministério da Saúde (BR), Secretaria de Ciência, Tecnologia e Insumos Estratégicos. Departamento de Assistência Farmacêutica. A fitoterapia no SUS e o programa de pesquisas de plantas medicinais da central de medicamentos, 9-28.

Santos, J. F., Pagani, E., Ramos, J. \& Rodrigues, E. (2012). Observations on the therapeutic practices of riverine communities of the Unini River, AM, Brazil. Journal of Ethnopharmacology. 142, 503-515.

Santos, M. C. \& Tesser, C. D. (2012). Um método para a implantação e promoção de acesso às Práticas Integrativas e Complementares na Atenção Primária à Saúde. Ciência \& Saúde Coletiva. 17, 3011-3024.

Santos, M. R. A., Lima, M. R. \& Oliveira, C. L. L. G. (2014). Medicinal plants used in Rondônia, Western Amazon, Brazil. Revista Brasileira de Plantas Medicinais. 16, 707-720.

Santos, S. S., Léda, P. H. O., \& Oliveira, D. R. (2018). Plantas Medicinais e Fitoterapia em Oriximiná - Pará, Brasil: Percepção e Intenção de Uso pelos Profissionais do Sistema Único de Saúde (SUS). Vittalle. 30, 11-25.

Telesi-Júnior, E. (2016). Práticas integrativas e complementares em saúde, uma nova eficácia para o SUS. Estudos Avançados. $30,99-112$.

Unesco (2020). Culture and Health. Orientation Texts: World Decade for Cultural Development, Document CLT/DEC/PRO, 129 p.

Vale, N. B. (2002). A farmacobotânica, ainda tem lugar na moderna anestesiologia? Revista Brasileira de Anestesiologia. 52, 368-80.

Vásquez, S. P., Mendonça, M. S. \& Noda, S. D. (2014). Etnobotânica de plantas medicinais em comunidades ribeirinhas do Município de Manacapuru, Amazonas, Brasil. Acta Amazonica. 44, 457-472.

Veiga, J. B. \& Scudeller, V. V. (2015). Etnobotânica e medicina popular no tratamento de malária e males associados na comunidade ribeirinha Julião - baixo Rio Negro (Amazônia Central). Revista Brasileira de Plantas Medicinais. 17, 737-747.

Who. (2018). Guidelines on Good Herbal Processing Practices for Herbal Medicines. WHO Technical Report Series, No. 1010 , Annex 1. 